Open Access

\title{
Statins induce insulin-degrading enzyme secretion from astrocytes via an autophagy-based unconventional secretory pathway
}

Sung Min Son ${ }^{1,2}$, Seokjo Kang ${ }^{1}$, Heesun Choi ${ }^{1}$ and Inhee Mook-Jung ${ }^{1,2^{*}}$

\begin{abstract}
Background: Insulin degrading enzyme (IDE) is a major protease of amyloid beta peptide (AB), a prominent toxic protein in Alzheimer's disease (AD) pathogenesis. Previous studies suggested that statins promote IDE secretion; however, the underlying mechanism is unknown, as IDE has no signal sequence.

Results: In this study, we found that simvastatin $\left(0.2 \mu \mathrm{M}\right.$ for $12 \mathrm{~h}$ ) induced the degradation of extracellular $A \beta_{40}$, which depended on IDE secretion from primary astrocytes. In addition, simvastatin increased IDE secretion from astrocytes in a time- and dose-dependent manner. Moreover, simvastatin-mediated IDE secretion was mediated by an autophagy-based unconventional secretory pathway, and autophagic flux regulated simvastatin-mediated IDE secretion. Finally, simvastatin activated autophagy via the LKB1-AMPK-mTOR signaling pathway in astrocytes.
\end{abstract}

Conclusions: These results demonstrate a novel pathway for statin-mediated IDE secretion from astrocytes. Modulation of this pathway could provide a potential therapeutic target for treatment of $A \beta$ pathology by enhancing extracellular clearance of $A \beta$.

Keywords: Statin, Insulin-degrading enzyme (IDE), Autophagy-based unconventional secretion, Amyloid- $\beta$ (A $\beta$ ), Alzheimer's disease (AD)

\section{Background}

Alzheimer's disease $(\mathrm{AD})$ is the most common form of dementia; it is characterized by senile plaques, neurofibrillary tangles, and neuronal cell death $[1,2]$. Abnormally increased levels of amyloid beta peptides $(A \beta)$ lead to formation of extracellular senile plaques and are associated with neurodegeneration in $\mathrm{AD}[3,4]$. The $\mathrm{A} \beta$ levels in the brain are not only determined by the rate of production by amyloid precursor protein (APP) processing $[5,6]$, but also by several clearance mechanisms. These include proteolytic degradation of extracellular $A \beta$ by cell surface-localized and/or secreted proteases such as neprilysin (NEP), matrix metalloproteinase-9 (MMP-9), and

\footnotetext{
* Correspondence: inhee@snu.ac.kr

'Department of Biochemistry \& Biomedical Sciences, Seoul National University College of Medicine, 103 Daehak-ro, Jongro-gu, Seoul 110-799, Korea

${ }^{2}$ Neuroscience Research Institute, Seoul National University College of Medicine, Seoul, Korea
}

insulin-degrading enzyme (IDE) [7-9]. NEP is located mainly in the plasma membrane, and its catalytic domain faces the extracellular space [7]. MMP-9 and IDE can be secreted extracellularly and degrade extracellular $A \beta$, despite the fact that IDE has no signal sequence for secretion through a conventional secretory pathway [10]. Many studies have demonstrated that IDE is secreted [11]; however, the mechanism of secretion is still elusive.

Macroautophagy (hereafter referred to as autophagy) is a fundamental biological process in eukaryotes and has an impact on essential biological processes including aging, cancer, neurodegenerative diseases, and metabolic disorders $[12,13]$. Autophagy is currently best known as a degradative pathway that delivers cytoplasmic materials and organelles to the lysosomes for degradation [14]. All autophagy-related processes include the formation of double-membrane structures called autophagosomes and are induced by the inhibition of the mammalian target 
of rapamycin (mTOR) signaling pathway. Autophagosomes and their contents undergo clearance upon fusion with an endosome (amphisomes) or lysosome (autolysosomes) for degradation and recycling (autophagic flux) [12, 13, 15]. However, recent studies show that autophagy also has a role in non-autophagic processes, especially in the secretory pathway [16]. In eukaryotic cells, the autophagy-based secretory pathway regulates the unconventional secretion of several cytosolic proteins or factors such as IL (interleukin)-1 $\beta$, IL-18, High-mobility Group Box 1 (HMGB1), ATP (adenosine triphosphate), $\mathrm{A} \beta$, and von Willebrand factor [17-20]. These proteins share important features, including the lack of a signal sequence for conventional secretion, and the contribution of autophagy-related (Atg) protein to their secretion.

Several studies report that increased cholesterol levels might be related to $\mathrm{AD}[21,22]$, and that statin-mediated inhibition of 3-hydroxy-3-methylglutaryl-coenzyme A (HMG-CoA) reductase decreases cholesterol levels; thus, reducing $A \beta$ levels $[23,24]$. However, this is controversial [22]. Several studies have demonstrated that statins can decrease the generation of $\mathrm{A} \beta$ by enhancing nonamyloidogenic processing of APP $[25,26]$. In addition, statins also impair the generation of isoprenoids, which play important roles in the post-translational modification of proteins in the Rho and Rab families [26, 27]. Isoprenoids regulate the localization and biological function of Rho and Rab proteins, and affect $A \beta$ generation by modulating APP processing [27]. Previous studies have also shown that statins promote $A \beta$ degradation by microglia via IDE secretion [24]. However, the molecular mechanisms by which statins could offer protection against AD have not been studied extensively.

In this study, we found that IDE secretion from primary astrocytes was increased by statins in a time- and concentration-dependent manner, and statin-induced IDE secretion was associated with autophagy-based unconventional secretion. Additionally, we found that autophagic flux is important in IDE secretion and that statin activates autophagy in astrocytes via the LKB1AMPK-mTOR signal pathway. These results indicate that IDE is secreted from astrocytes via an autophagybased secretory pathway, and that regulation of autophagy is a potential therapeutic target in $A \beta$ pathology.

\section{Results}

Statins induce extracellular secretion of functional IDE from astrocytes

Previous studies have shown that astrocytes are the main source for IDE in AD pathology [28]. Therefore, we first determined whether statins regulate IDE levels in the extracellular space of astrocytes. We found that simvastatin increased IDE secretion from primary astrocytes in a time- and dose-dependent manner (Fig. 1a-c), and that IDE levels in the cells were reduced conversely (Fig. 1a, b). Because statins are known to lower cholesterol levels, we checked whether statins regulate cholesterol levels in astrocytes. By using a filipin assay, we found that simvastatin reduced intracellular cholesterol levels (Additional file 1: Figure S1A,B). To determine whether only simvastatin induced IDE secretion from astrocytes, one of the other known statins, fluvastatin, was applied to astrocytes; we found that fluvastatin also increased IDE secretion (Additional file 2: Figure S2A,B). To examine whether the IDE secreted by statin treatment functions as insulysin (having an insulin-degrading function), we utilized an IDE enzymatic activity assay. We detected an increased in fluorescent intensity generated by the cleavage of fluorometric IDE substrates in the statin-treated astrocyte-conditioned media (ACM) (Fig. 1d). In an alternative approach, we performed an $A \beta$ degradation assay. When the statin-treated ACM was incubated with the recombinant $A \beta_{40}$ peptide, the level of the remaining $A \beta_{40}$ peptide was reduced (Fig. 1e; lane 1 vs. lane 2). Furthermore, the reduced $A \beta_{40}$ levels after incubation with the statin-treated ACM were restored when bacitracin A, an IDE inhibitor, was added (Fig. 1e; lane 2 vs. lane 6). The inhibitors of other known A $\beta$ degrading enzymes including thiorphan, a NEP inhibitor, or GM6001, a MMP inhibitor, did not restore the reduced $A \beta_{40}$ levels by the statin. These data demonstrate that statins induce IDE secretion from astrocytes, and statin-induced IDE functions as a protease to degrade the $A \beta$ peptide.

\section{Statin-mediated IDE secretion from astrocytes is associated with an autophagy-based unconventional secretory pathway}

To examine the mechanism of statin-induced IDE secretion, transcript levels of IDE after statin treatment were measured by quantitative real-time PCR (qPCR). Simvastatin did not alter ide mRNA levels in astrocytes (Fig. 2a). Because IDE has no signal sequence for secretion, we further investigated the secretory mechanism of IDE. Previous studies showed that statins could stimulate secretion of IDE proteins via an unconventional pathway in association with exosomes [24]. To determine whether statin-induced IDE secretion is associated with exosomes in astrocytes, both exosomes and non-exosome fractions were isolated from vehicle- or statin-treated ACM. We found that simvastatin increased secreted IDE levels both in the exosomes and non-exosome fractions (Additional file 3: Figure S3), indicating that the secretory pathway for IDE is mediated by both exosome- and nonexosome-associated pathways. To determine the exact mechanisms of IDE secretion by statin, we focused on the autophagy-based unconventional secretory pathway. We used immunostaining and western blot analysis and found 


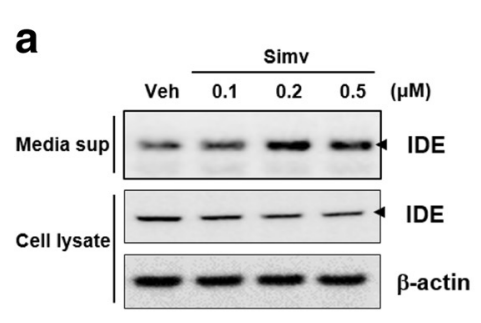

b

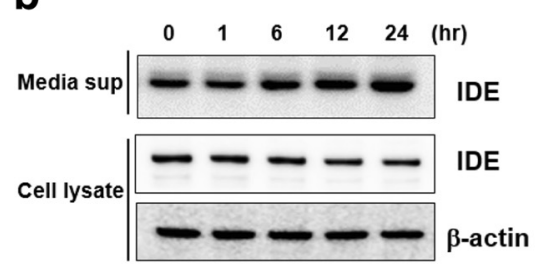

$\square$ Media sup

Cell lysate
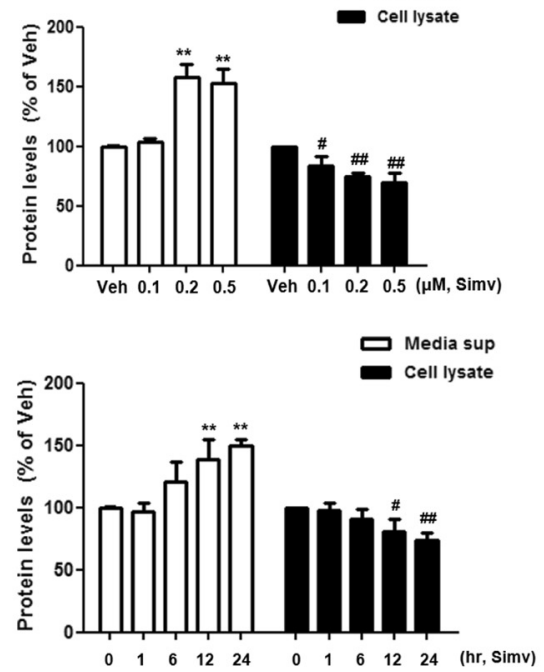

C

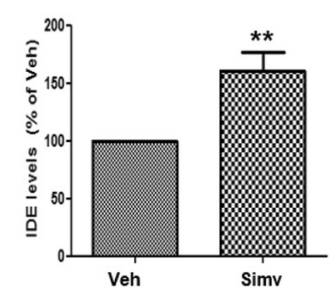

d

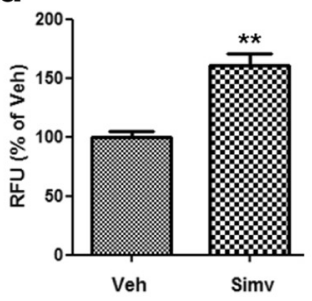

e

\begin{tabular}{|c|c|c|c|c|c|c|c|}
\hline Media & : & Veh & 0.2 & 0.2 & 0.2 & 0.2 & $0.2(\mu \mathrm{M}$, Simv. \\
\hline $\mathrm{A} \beta 40(1 \mu \mathrm{M})$ & : & + & + & + & + & + & + \\
\hline rIDE $(0.3 \mu \mathrm{g} / \mathrm{ul})$ & ) : & - & - & + & - & - & - \\
\hline 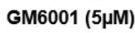 & ): & - & - & - & + & - & - \\
\hline Thio $(5 \mu \mathrm{M})$ & : & - & - & - & - & + & - \\
\hline Baci. A $(20 \mu M)$ & 1) : & - & - & - & - & - & + \\
\hline
\end{tabular}

f

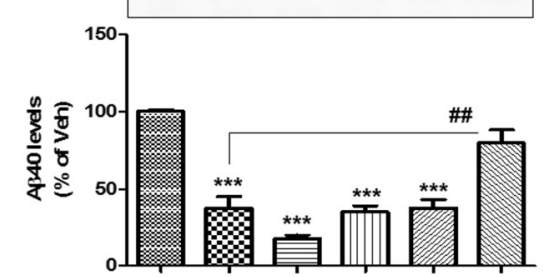

Fig. 1 Statin treatment induces extracellular secretion of functional insulin-degrading enzyme (IDE) from astrocytes. a \& b Increased IDE levels secreted from the primary astrocytes by simvastatin in a concentration-(a) and time-(b) dependent manner. The time points mean the time period of simvastatin treatment. Blots are representative of at least three independent experiments ( $N=3$ experiments). ${ }^{* *} p<0.01, \# p<0.05$, \#\# $p<0.01$ vs. vehicle-treated cells. c Secreted IDE levels by simvastatin with IDE ELISA. d IDE enzymatic activity in the media from simvastatin-treated cells. (e \& f) Cell-free $A \beta$ degradation assay. The arrowhead indicates remaining $A \beta_{40}$ levels. Data were obtained from at least three replicates for each group ( $N=3$ experiments). rIDE, recombinant IDE protein; Thio, thiorphan; Baci. A, Bacitracin A. ${ }^{* *} p<0.01,{ }^{* * *} p<0.001$ vs. vehicle-treated cells; \#\# $p<0.01$ vs. cells treated with simvastatin

that statin treatment activated autophagy (Fig. 2b,c). In addition, we found that statin-induced IDE secretion was blocked by autophagy inhibition (Fig. 2c-e). Treatment with 3-methyladenine (3MA), a well-known autophagy inhibitor (Fig. 2c,d), or the knock-down of Beclin1, a key component of autophagy-initiation complex, reduced statin-induced IDE secretion from astrocytes (Fig. 2e). In the IDE enzymatic assay, 3MA inhibited increased IDE activity by statin treatment (Fig. 2f). To visualize whether IDE exists in the autophagosomes, IDE and LC3 were stained with their specific antibodies, followed by observation using confocal microscopy. Immunocytochemistry data showed that IDE was located in the autophagosomes, and simvastatin increased the level of IDE in the autophagosomes (Fig. 2g,h). Taken together, these data imply that statininduced IDE secretion is tightly regulated by the autophagy pathway in astrocytes.

\section{Statin-mediated IDE secretion from astrocytes is regulated by autophagic flux}

Previous studies showed that secretion of IL- $1 \beta$, one of the substrates for autophagy-based unconventional secretion, 
a

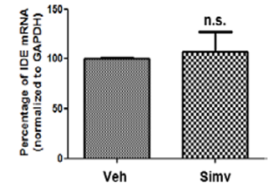

C

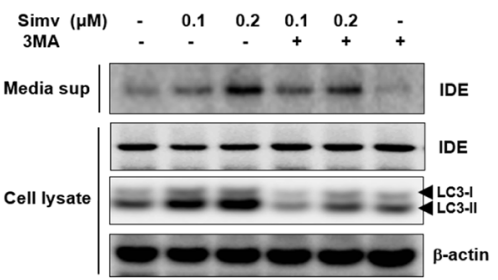

e

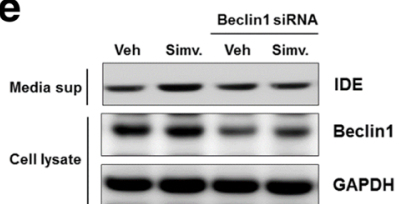

b

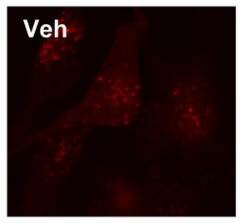

d

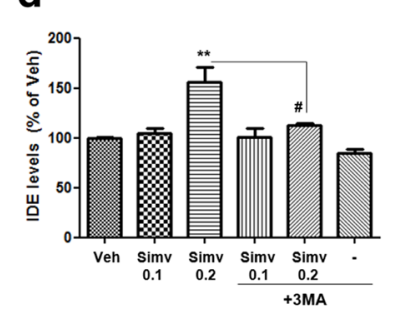

f
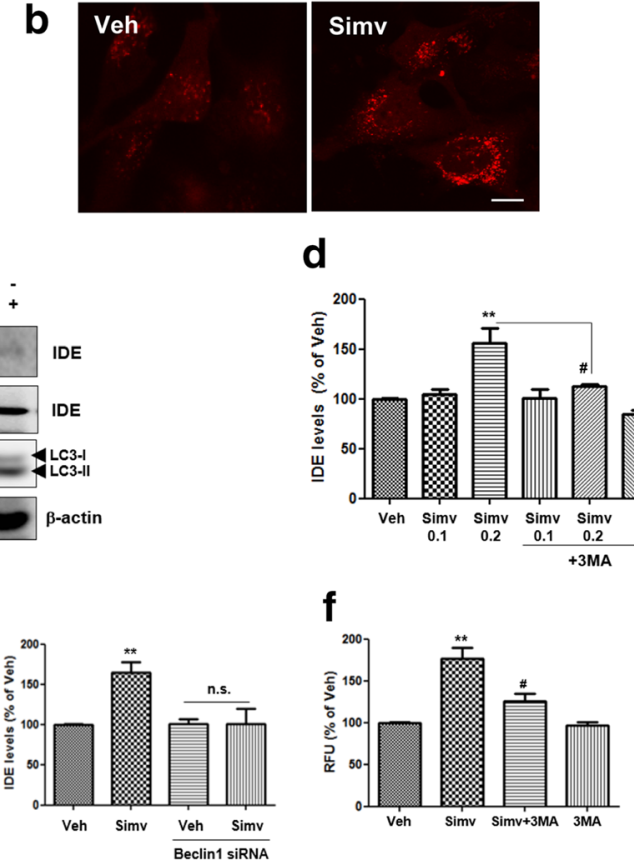

\section{.}

\section{g}



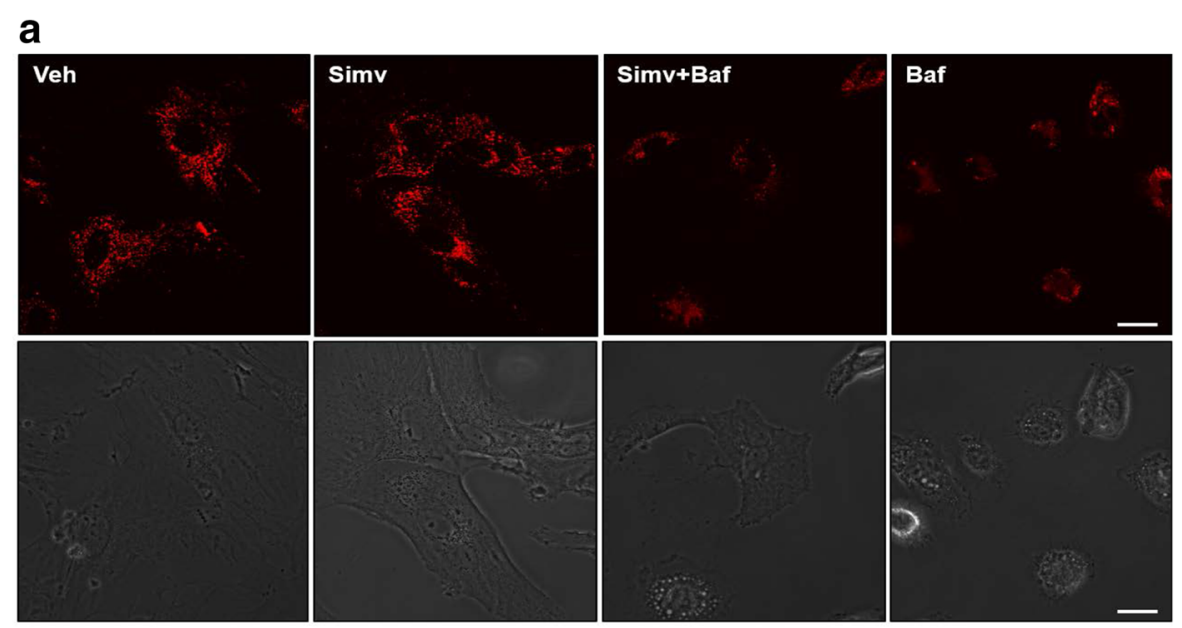

b
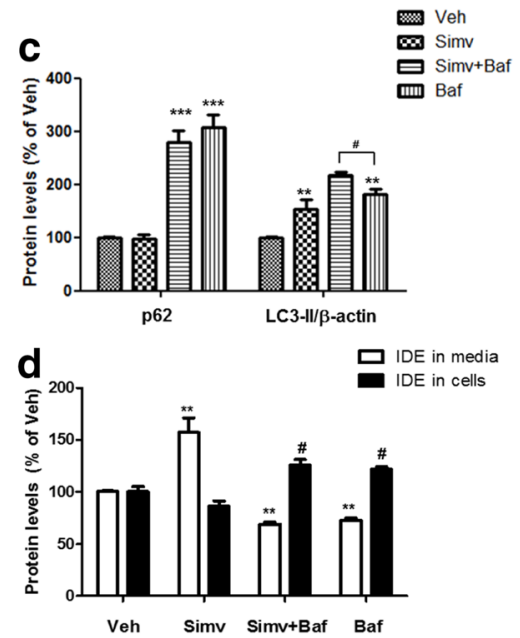

Fig. 3 Statin-mediated IDE secretion from astrocytes is regulated by autophagic flux. a Lysosomal activity as determined with LysoTracker probe. Scale bar represents $10 \mu \mathrm{m}$. b Change in secreted IDE levels by inhibiting lysosome with bafilomycin. c \& $\mathbf{d}$ Quantitative analysis of Fig. $3 \mathrm{~b}$ ( $N=3$ experiments). Baf, bafilomycin. ${ }^{* *} p<0.01$, \# $p<0.05$ vs. vehicle-treated cells

observed that statin treatment decreased the phosphorylation of mTOR, and increased LC3-II levels (Fig. 4a, b). Conversely, compound $\mathrm{C}$ reversed these changes in mTOR kinase and LC3-II levels by statins (Fig. 4a, b). In addition, compound $C$ inhibited statin-induced IDE secretion significantly (Fig. 4a, b). To visualize the alterations in autophagosome formation caused by the AMPK-specific inhibitor directly, immunocytochemistry was performed after co-treatment with compound $\mathrm{C}$ and statin. Statin treatment alone increased the punctate signal of LC3 (Fig. 4c), indicating enhanced autophagosome formation. Compound $\mathrm{C}$ prevented this increase, suggesting that AMPK signaling is required for statin treatment to promote autophagosome formation in astrocytes.

\section{Simvastatin does not regulate MMP-9 and NEP levels in} astrocytes

In above data, simvastatin activated autophagy via AMPK-mTOR pathway, and statin-induced autophagy activation is neccessary for IDE secretion. To investigate whether other $\mathrm{A} \beta$ degradation enzymes such as MMP-9 and NEP can be also regulated by autophagy, MMP-9 and NEP levels were measured with media and cell lysates under simvastatin and/or Compound $\mathrm{C}$ treated condition. We found that simvastatin did not alter MMP-9 and NEP levels (Fig. 4d and e).

\section{LKB1, but not CaMKK $\beta$, mediates the statin-mediated phosphorylation of AMPK}

In previous studies, 2 upstream kinases were reported to activate AMPK, liver kinase B1 (LKB1) and $\mathrm{Ca}^{2+} / \mathrm{cal}-$ modulin-dependent protein kinase kinase-beta $(\mathrm{CaMKK} \beta)$ $[32,33]$. We found that statin treatment induced LKB1 phosphorylation (Fig. 5a), and LKB1-specific siRNA failed to induce statin-induced phosphorylation of AMPK or generation of LC3-II (Fig. 5b-d), indicating that LKB1 is an AMPK upstream kinase for statin-induced autophagosome formation in astrocytes. When CaMKK $\beta$-specific 


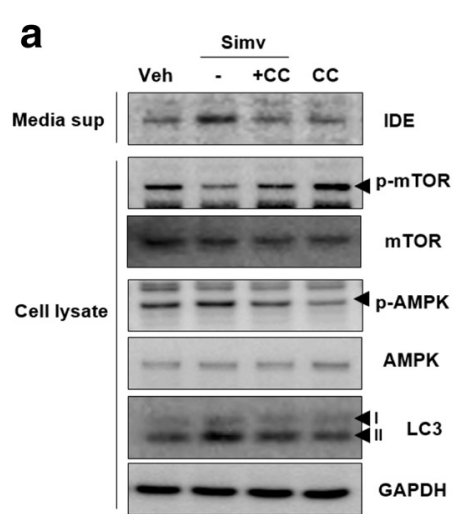

C
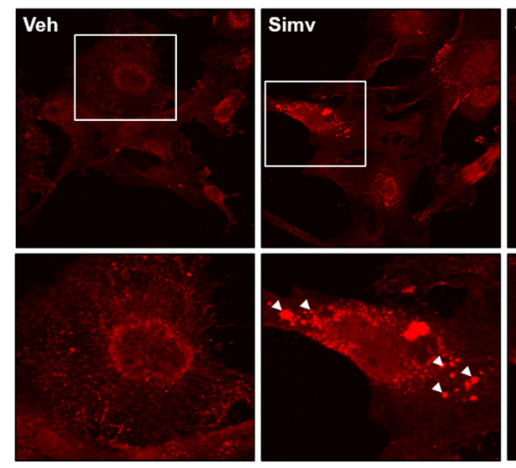

d

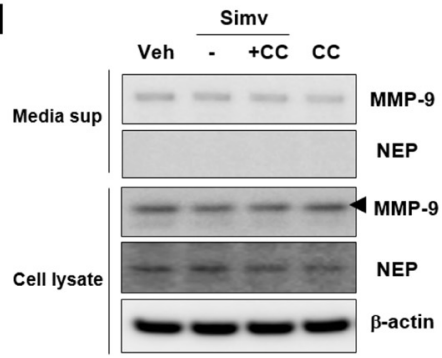

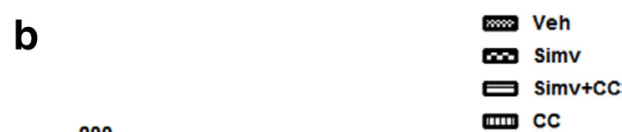
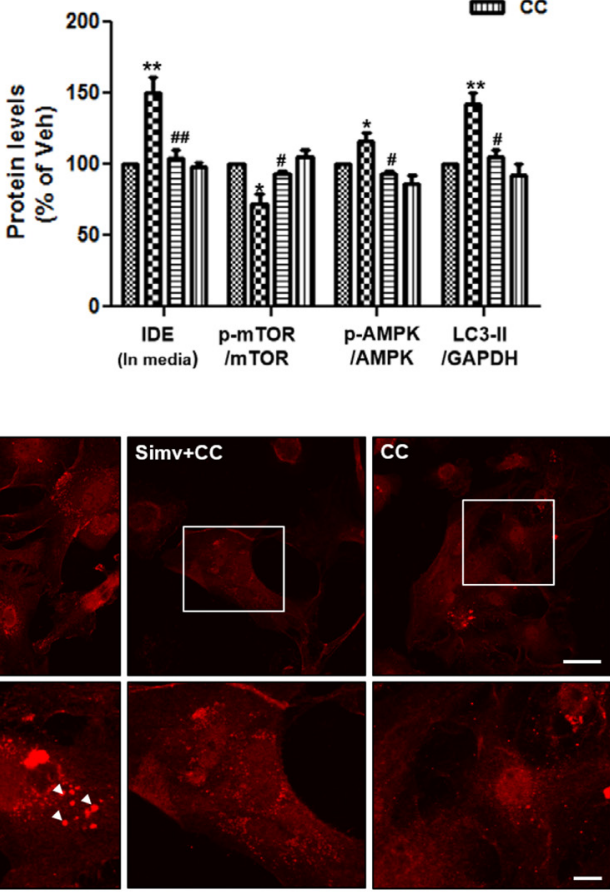

e

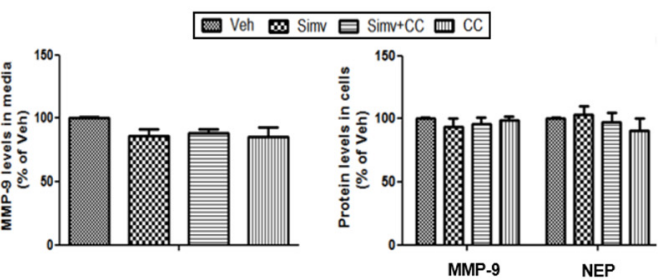

Fig. 4 Statin activates autophagy via AMPK-mTOR signaling pathway in astrocytes. a Inhibition of statin-mediated autophagy activation and IDE secretion by treatment with AMPK inhibitor (Compound C; CC). b Quantitative analysis of Fig. $4 a$ ( $N=3$ experiments). ${ }^{*} p<0.05,{ }^{* *} p<0.01$ vs. vehicle-treated cells; \# $p<0.05$, \#\# $p<0.01$ vs. cells treated with simvastatin. $\mathbf{c}$ Inhibition of statin-mediated autophagy activation by treatment with compound C. Anti-LC3 antibody was used to detect autophagy. Scale bar represents $6 \mu \mathrm{m}$. Lower panels show figures under higher magnification. Scale bar represents $2 \mu \mathrm{m}$. $\mathbf{d}$ The effect of statins on regulation of MMP-9 and NEP levels. e Quantitative analysis of Fig. $4 \mathrm{~d}$ ( $N=3$ experiments)

siRNA was transfected into astrocytes, statin-induced phosphorylation of AMPK and levels of LC3-II were not altered (Fig. 5b-d), indicating that CaMKK $\beta$ is not required for statin-induced autophagosome formation in astrocytes. These data imply that LKB1, not CaMKK $\beta$, mediates statin-induced AMPK phosphorylation and then induces autophagosome formation.

\section{Discussion}

In this study, we first demonstrated that statin treatment could induce extracellular IDE secretion from astrocytes via an autophagy-based unconventional secretory pathway (Fig. 6). Secreted IDE can significantly degrade extracellular
$\mathrm{A} \beta$ peptides, indicating that it may be important for $\mathrm{AD}$ progression. In microglia, IDE, which has no known signal sequences, is secreted under statin-treated conditions via an exosome associated unconventional secretory pathway [24]. To determine whether statin-induced IDE secretion is associated with exosomes in astrocytes, both exosomes and non-exosome fractions were isolated from vehicle- or statin-treated astrocyte-conditioned media (ACM). We found that statin application increased IDE secretion from both the exosome and non-exosome fractions (Additional file 3: Figure S3), indicating that the secretory pathway for IDE is mediated by both exosome- and non-exosomeassociated pathways. We also found that statin treatment 

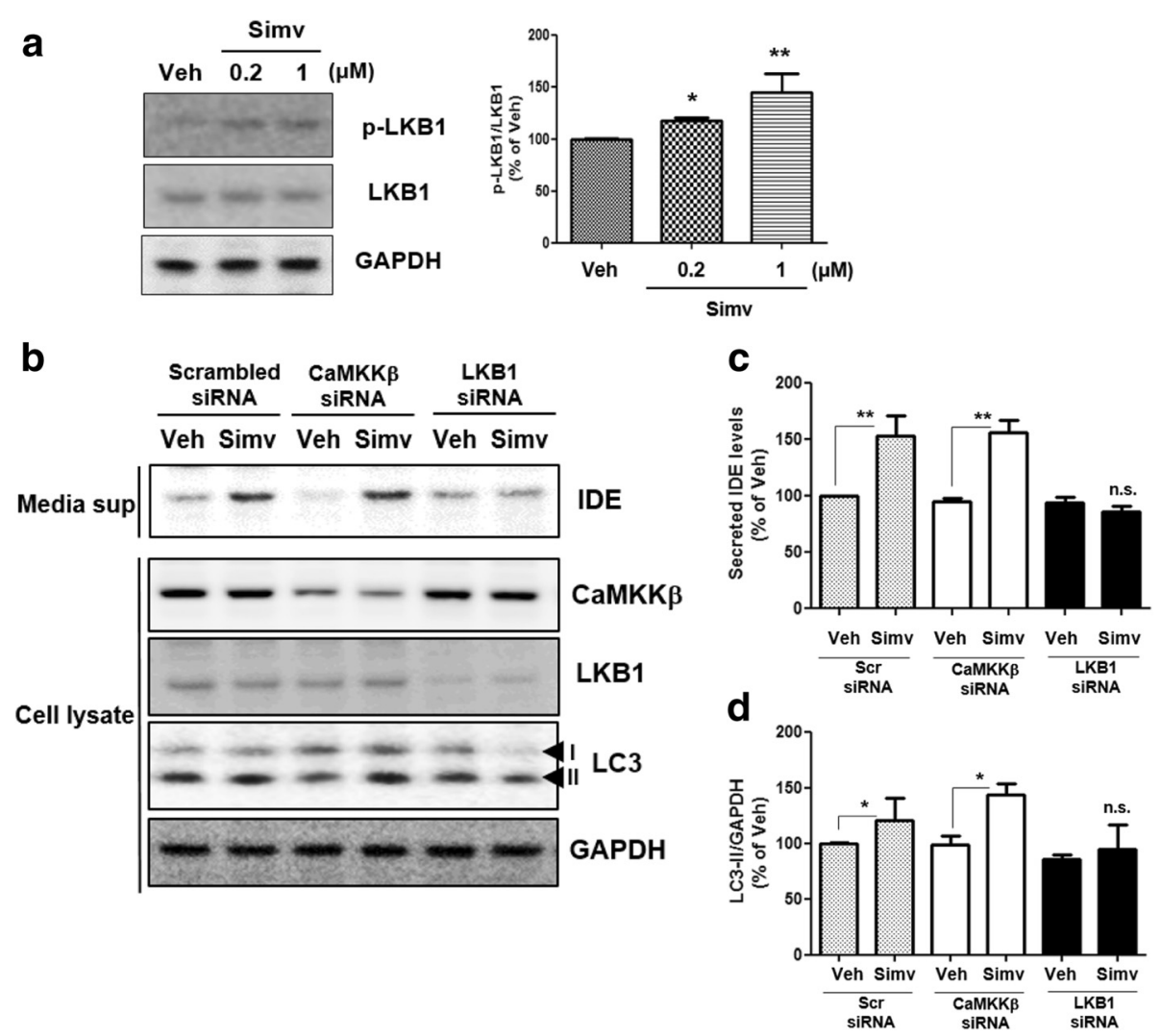

Fig. 5 Statin-induced AMPK activation is mediated by LKB1 in astrocytes. a Increased phosphorylation of LKB1 by treatment with simvastatin. $\mathbf{b}$ Statin-induced autophagy activation and IDE secretion levels in CaMKKß or LKB1 knock-downed cells. $\mathbf{c}$ \& $\mathbf{d}$ Quantitative analysis of Fig. 5b ( $N=3$ experiments). ${ }^{*} p<0.05,{ }^{* *} p<0.01$ vs. vehicle-treated cells. n.s. indicates no significant difference

increased functional IDE secretion in a time- and dosedependent manner, and statin-induced IDE secretion was blocked by autophagy inhibition although IDE secretion at basal condition might be mediated by an autophagyindependent pathway (Fig. 2d-f). Because the fundamental role of autophagy is the clearance of protein aggregates and pathogens [13], we determined whether statinmediated IDE secretion is regulated by the autophagylysosome pathway. When lysosomes were disrupted by the lysosomal inhibitor bafilomycin, IDE secretion was blocked, indicating that autophagic flux is important for statin-mediated IDE secretion. In this study, cell death was not observed under several drug-treated conditions (Additional file 4: Figure S4).

Astrocytes have an important role in maintaining neuronal homeostasis by providing energy and eliminating waste [34]. In addition, astrocytes are well positioned both metabolically and anatomically to play an important homeostatic role under basal conditions as well as pathological conditions. Previous studies have shown that at an early stage of $\mathrm{AD}$, activated astrocytes surround and infiltrate $A \beta$ plaque $[35,36]$. Although their exact role in $\mathrm{AD}$ pathogenesis remains unknown, reactive astrocytes have been shown to play a role in the clearance of $A \beta$ suggesting a neuroprotective role in $A D$ [37]. For the removal of extracellular $A \beta$, astrocytes take up $A \beta$ bound to membrane receptors, such as LRP1, via endocytosis and degrade $A \beta$ [38]. In addition, astrocytes are capable of degrading $A \beta$ enzymatically by secretion of MMP-9 and IDE extracellularly [39, 40]. Especially, in AD pathology, astrocytes are the main source for IDE [28]. Our results provide evidence that in statin-treated conditions, astrocytes can release IDE extracellularly via an autophagy-based secretory pathway, and the secreted IDE could then degrade extracellular $A \beta$. To determine whether secretion of other $A \beta$ degrading enzymes such as NEP or MMP-9 is induced by autophagy similarly to IDE, we found that simvastatin did not induce secretion of NEP and MMP-9 (Fig. 4d and e). Previous studies have indicated that NEP is located mainly in the plasma membrane [7], and secretion of MMP-9 is mediated through a conventional secretory pathway, as MMP-9 has signal peptides for secretion [9]. Therefore, among $A \beta$-degrading enzymes, only IDE may be secreted by an autophagy-based secretory pathway.

There are many reports that elevated levels of cholesterol increase the risk for $\mathrm{AD}$ and that statins can regulate $\mathrm{AD}$ progression [21, 22]. Several studies have 


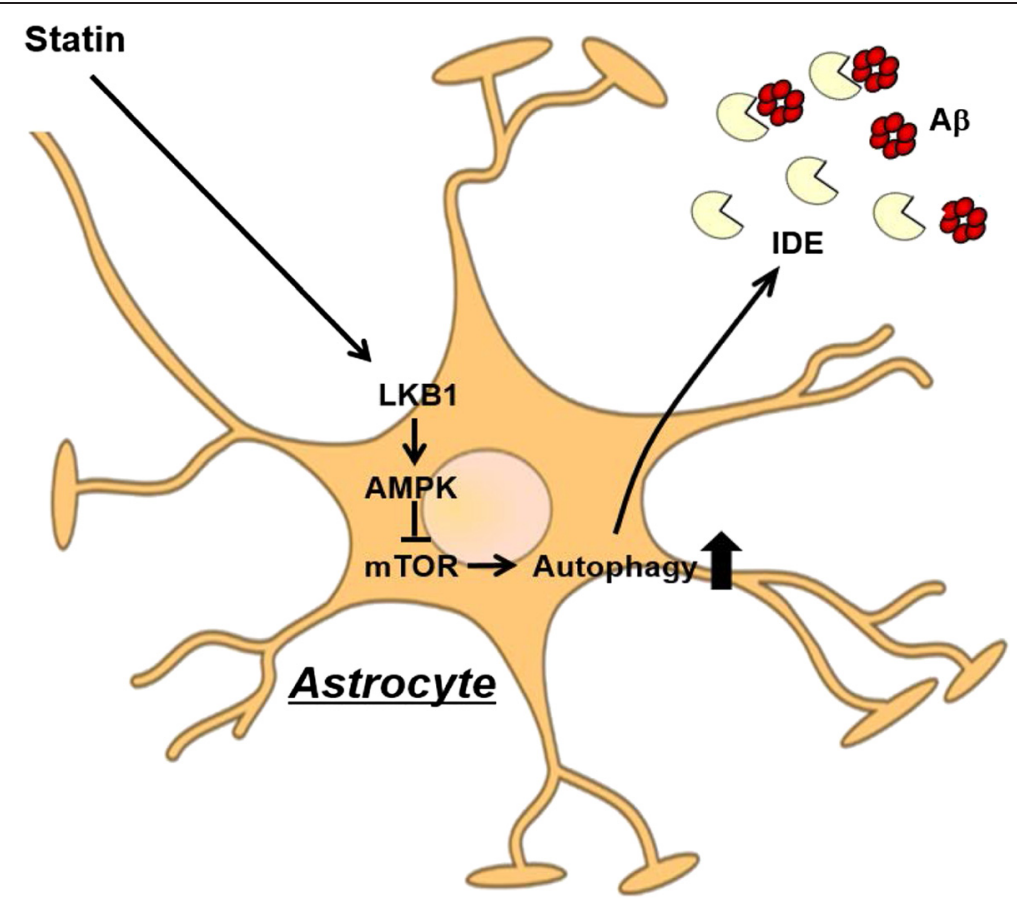

Fig. 6 Schematic diagram. Statins activate autophagy via the LKB1-AMPK-mTOR signaling pathway in astrocytes, and statin-mediated IDE secretion is mediated by an autophagy-based unconventional secretory pathway. Finally, secreted IDE can significantly decrease in extracellular A $\beta$ levels, thus, modulation of this pathway can regulate AD progression by enhancing $A \beta$ clearance

reported beneficial effects of statin treatment on $\mathrm{AD}$ pathology [24, 41]. However, some studies did not report any positive effects of statin; statin treatment actually increased $A \beta$ levels $[22,42]$. Such variable conclusions might stem from the fact that most of these studies were conducted with randomized concentrations of statins and time of statin treatment (early stage or late stage of $\mathrm{AD})$ and the differential ability of statins to cross the blood-brain barrier (BBB) [24]. In our data, lysosomal dysfunction by treatment with bafilomycin inhibited statin-mediated IDE secretion. Because late-stage AD brains show autophagy accumulation and lysosomal dysfunction [43], it is possible that statins may not be beneficial when administered to patients with latestage $\mathrm{AD}$.

Abnormally pathological autophagic vacuoles (AVs) accumulate in the brains of patients with $\mathrm{AD}$ and in brains from animal models of $\mathrm{AD}$ [44]. The excessive autophagosome formation might result from increased induction via the AMPK-mTOR pathway and dysfunction in autophagy-lysosomal degradation [31, 43]. Because maintaining autophagic flux is important for cell survival [13], dysregulated autophagy could accelerate $\mathrm{AD}$ progression; however, the mechanism is not yet fully understood. In this study, impaired autophagic flux inhibited statin-mediated IDE secretion from astrocytes; the reduced IDE secretion from astrocytes could lead to an increase in extracellular $A \beta$ levels. The regulation of
$A \beta$ levels is of great interest for $A D$ treatment. Apart from the interference with $A \beta$ generation, a promising alternative may be the enhancement of $A \beta$ degradation by targeting $A \beta$-degrading enzymes [45]. Thus, regulating IDE secretion by statins in astrocytes may lead to new therapeutic approaches for sporadic AD.

\section{Conclusions}

Our data collectively suggest that statin treatment can induce extracellular IDE secretion from astrocytes via an autophagy-based unconventional secretory pathway. Because secreted IDE can significantly decrease in extracellular $A \beta$ levels, modulation of this pathway could provide a potential therapeutic target for treatment of $A \beta$ pathology.

\section{Methods}

Cell cultures, drug treatments, and transfection

Primary astrocytes were prepared from newborn (P1) ICR mice as described previously [46]. The cells underwent two passages for the experiments and were grown in DMEM supplemented with $10 \%$ fetal bovine serum (FBS; HyClone, Irvine, CA, USA) and $0.1 \mathrm{mg} / \mathrm{ml} \mathrm{P/S}$ (Penicillin-Streptomycin; Sigma-Aldrich, St. Louis, MO, USA) at $37{ }^{\circ} \mathrm{C}$ in humidified $5 \% \mathrm{CO}_{2}$ incubator for 2 weeks. The cells were treated with several reagents alone, or co-treated with simvastatin followed by $24 \mathrm{~h}$ incubation. Reagents used in this study were thiorphan 
(T6031; NEP inhibitor), bacitracin A (B0125; IDE inhibitor), 3MA (M9281; autophagy blocker), bafilomycin (B1793; selective inhibitor of vacuolar-type $\mathrm{H}^{+}$-ATPase), simvastatin (S6196), fluvastatin (SML0038), STO-609 (S1318; CaMKK $\beta$ inhibitor), methyl- $\beta$-cyclodextrin (M $\beta C D$ ) (C4555; cholesterol-lowering compound) from Sigma-Aldrich; compound C (171260; AMPK inhibitor) from Calbiochem (San Diego, CA, USA); GM6001 (142880-36-2; MMP inhibitor) from Tocris Bioscience (Bristol, UK). The siRNAs were purchased from Bioneer Inc. (Daejeon, Korea) (1330628 for beclin 1, 1432200 for $l k b 1,1334167$ for camkk2), and were transfected into the cells using RNAimax (13778) (Invitrogen, Carlsbad, CA) according to the manufacturer's instructions.

\section{Western blot analysis}

Harvested cell pellets and media were prepared as described previously [47]. Briefly, cell pellets were lysed in RIPA buffer (50 mM Tris-Cl, pH 8.0, $150 \mathrm{mM} \mathrm{NaCl}, 1 \%$ NP-40, $0.5 \%$ NaDoc, $0.1 \%$ SDS) containing protease inhibitors (Sigma-Aldrich). After sonication at $4{ }^{\circ} \mathrm{C}, 10 \mu \mathrm{g}$ of lysate was separated on SDS-PAGE gels and then transferred to PVDF membranes. Membranes were incubated with antibodies against the indicated proteins in this study. The antibodies for the western blot analysis were: anti-LC3B antibody (1:2,000; M152-3, MBL, Woburn, MA, USA; 2775, Cell Signaling Technology, Beverly, MA, USA); 6E10 (1:5000; SIG-39300, Covance, Princeton, NJ, USA); anti-p-LKB1 (S428), anti-AMPK (2532), anti-pAMPK (T172) (2535) and anti-p-mTOR (S2448) (5536) (1:2000, Cell Signaling Technology); anti-LKB1 (sc-32245), anti-IDE (N-15) (sc-27265) and anti-mTOR (sc-1549) (1:1,000; Santa Cruz Biotechnologies Inc., Santa Cruz, CA, USA); anti-IDE (ab32216), anti-GAPDH (ab9485), anti-TSG101 (ab83) and anti-beclin1 (ab62472) (1:2,000; Abcam, Cambridge, MA, USA); anti-p62 (P0067), and anti- $\beta$-actin (A1978) (1:4,000; SigmaAldrich). Immunoreactivity was determined by chemiluminescence (GE Healthcare, Piscataway, NJ, USA). The chemiluminescence signal was detected with a digital image analyzer (LAS-3000; Fuji, Tokyo, Japan).

\section{$A \beta$ degradation assay}

The $A \beta$ degradation assay was performed as previously described with modification [24]. Briefly, primary astrocytes grown in a 6-well plate were incubated with simvastatin or DMSO (vehicle) in serum-free DMEM for $24 \mathrm{~h}$. The supernatant was then collected after centrifugation and incubated with $1 \mu \mathrm{M}$ recombinant human $A \beta_{40}$ (American Peptide, Sunnyvale, CA, USA) for $12 \mathrm{~h}$ at $37{ }^{\circ} \mathrm{C}$ in the absence or presence of $5 \mu \mathrm{M}$ GM6001, $5 \mu \mathrm{M}$ thiorphan, $20 \mu \mathrm{M}$ bacitracin A or $0.3 \mu \mathrm{g} / \mu \mathrm{l}$ recombinant IDE (rIDE). Remaining $A \beta_{40}$ levels were quantified with 6E10 antibody by western blot analysis.

\section{Trichloroacetic acid (TCA) precipitation}

For analyzing protein in the medium, we performed TCA precipitation as previously described [48]. Briefly, cell medium was centrifuged at 2,400 g for $5 \mathrm{~min}$ to remove cell debris and then subjected to TCA precipitation (up to $10 \%$ ) (T6399, Sigma-Aldrich).

\section{IDE activity assay and IDE level measurement in the media}

The IDE enzymatic activity in media was determined per manufacturer's protocol (CBA079, Calbiochem). Briefly, $50 \mu \mathrm{l}$ of the concentrated media were loaded into in a 96-well plate, which contained an affinity-purified polyclonal antibody that recognizes IDE. The media were concentrated with an Amicon Ultra filter (UFC510024, Millipore, Billerica, MA). Following $1 \mathrm{~h}$ incubation, fluorometric IDE substrates were added and incubated for $2-4 \mathrm{~h}$ at $37{ }^{\circ} \mathrm{C}$ in the dark. The fluorescence was measured using an excitation wavelength of $320 \mathrm{~nm}$ and an emission wavelength of $405 \mathrm{~nm}$. To determine the level of IDE in media, ELISA was performed per manufacturer's protocol (MBS725082, Mybiosource, San Diego, CA, USA).

\section{Measurement of lysosomal activity}

The measurement of lysosomal activity with LysoTrackerRed (L7528; Invitrogen) was performed as per manufacturer's protocol. The fluorescence intensity was observed using a confocal laser scanning microscope (FV10i-w, Olympus, Tokyo, Japan) and representative cells were selected and photographed.

\section{Quantitative real-time PCR (qRT-PCR)}

To examine the levels of IDE mRNA, qRT-PCR was performed as previously described [49]. RNA was isolated using the RNeasyPlus Mini Kit (Qiagen, Valencia, CA, USA) and cDNA was generated using the RevertAid First Strand cDNA Synthesis Kit (Fermentas, Glen Burnie, MD). Real-time PCR was performed on the cDNA samples using ABI StepOne 2.1 (Applied Biosystems, Foster City, CA), and the following sense and antisense primers were used: 5'-CCGGCCATCCAGAG AATAG AA-3' (sense), 5' - ACGGTATTCCCGTTTGTCTTCA3' (antisense) for IDE.

\section{Isolation and characterization of exosomes}

Exosomes and non-exosome fractions in the media from astrocytes were prepared as described earlier [50, 51]. In brief, primary astrocytes from T175 flasks (four flasks per one group) were cultured in DMEM with $10 \%$ FBS. One day before the exosome preparation, culture medium was replaced to AIM-V medium w/ or without simvastatin. Culture supernatants of cells grown for $24 \mathrm{~h}$ in AIM-V medium were collected and spun at $300 \mathrm{~g}$ for 
$10 \mathrm{~min}$ to remove cells. The supernatants were then sequentially centrifuged at $1,200 \mathrm{~g}$ for $20 \mathrm{~min}, 10,000 \mathrm{~g}$ for $30 \mathrm{~min}$, and 100,000 $\mathrm{g}$ for $1 \mathrm{~h}$. The 100,000 g pellet was washed, and then again spun at 100,000 g for $1 \mathrm{~h}$. The second 100,000 g pellet (exosomal pellet) was resuspended in PBS, and the supernatants were used as nonexosome fractions.

\section{Immunostaining}

Immunocytochemical staining was performed as described previously $[48,52]$. Briefly, the fixed cells were incubated with mouse anti-IDE (1:300) and/or anti-LC3B (1:300) primary antibodies in PBST (PBS with $0.2 \%$ Triton X-100) buffer overnight at $4{ }^{\circ} \mathrm{C}$. After several washes, the cells were incubated with secondary antibody, and images were taken using a confocal laser scanning microscope (FV10i-w; Olympus).

\section{Filipin staining}

The filipin staining was determined as manufacturer's protocol (F9765, Sigma-Aldrich). Briefly, the cells were fixed with $4 \%$ paraformaldehyde (PFA), and then were incubated with $25 \mu \mathrm{g} / \mathrm{ml}$ filipin in PBS for $30 \mathrm{~min}$ at room temperature. The images were taken using a confocal laser scanning microscope (FV10i-w; Olympus).

\section{Cell viability assay}

To measure cell viability, we used MTS assay kit (\#G3580, Promega, Madison, WI) according to the manufacturer's instructions. In Brief, the cells in a 96-well microtiter plate were incubated in the absence or presence of various drugs. After $24 \mathrm{~h}$ incubation, we transferred an appropriate volume of MTS assay solution into 96-well plate and incubated the plate for $1 \mathrm{~h}$ at $37^{\circ} \mathrm{C}$ in the dark. The absorbance was measured using a plate reader at $490 \mathrm{~nm}$.

\section{Statistical analysis}

For western blots, protein levels were normalized to pan forms or a housekeeping protein, such as $\beta$-actin or GAPDH. All data were expressed as means \pm standard error of the mean (S.E.M.). Statistical analysis was performed using GraphPad Prism 5 (San Diego, CA, USA). The data were analyzed by one-way analysis of variance with post-hoc test or unpaired $t$-tested regarded as appropriate. $P$ values of $<0.05$ were considered statistically significant.

\section{Additional files}

Additional file 1: Figure S1. Statins regulate cholesterol levels in astrocytes. (A) Cellular cholesterol levels were measured by filipin staining. $M \beta C D$ is a positive control. (B) Quantitative analysis of Figure
S1A using the Image J program ( $N=3$ experiments). ${ }^{* *} p<0.01,{ }^{* * *}$ $p<0.001$ vs. vehicle-treated cells. (PDF $217 \mathrm{~kb}$ )

Additional file 2: Figure S2. Fluvastatin induces IDE secretion from astrocytes. (A) Increased IDE levels secreted from the primary astrocytes by fluvastatin in a concentration-dependent manner. Blots are representative of at least 3 independent experiments ( $N=3$ experiments). (B) Quantitative analysis of Figure S2A. ${ }^{* *} p<0.01$ vs. vehicle-treated cells. (PDF 71 kb)

Additional file 3: Figure S3. Statin-induced IDE secretion is mediated by both exosome- and non-exosome-dependent pathways. (A) Western blot analysis of IDE levels in the exosomes or non-exosome fractions. TSG101 is an exosome marker protein. (PDF $30 \mathrm{~kb}$ )

Additional file 4: Figure S4. Cell death was not induced in this study. (A) MTS assay was used for checking cell viability under simvastatin, 3MA and/or bafilomycin treated condition. $N=5$ experiments. (PDF $57 \mathrm{~kb}$ )

\section{Abbreviations}

3-MA: 3-methyladenine; ACM: astrocyte-conditioned media; AD: Alzheimer's disease; APP: amyloid beta precursor protein; Atg: autophagy-related;

ATP: adenosine triphosphate; AVs: autophagic vacuoles; $A \beta$ : amyloid beta; Baf: bafilomycin; BBB: blood-brain barrier; CaMKKB: $\mathrm{Ca}^{2+} /$ calmodulindependent protein kinase kinase-beta; DMEM: Dulbecco's modified Eagle's medium; ELISA: enzyme-linked immunosorbent assay; EM: electron microscopy; FBS: fetal bovine serum; GAPDH: glyceraldehyde 3-phosphate dehydrogenase; HMGB1: high-mobility Group Box 1; IDE: insulin-degrading enzyme; LC3: microtubule-associated protein 1A/1B-light chain 3; LKB1: liver kinase B1; MMP-9: matrix metalloproteinase-9; mTOR: mammalian target of rapamycin; PBS: phosphate buffer saline; PFA: paraformaldehyde; qPCR: quantitative real-time polymerase chain reaction; TCA: Trichloroacetic acid; WB: western blotting.

\section{Competing interests}

The authors have declared that no conflict of interest exists.

\section{Authors' contributions}

S.M.S. designed and performed the experiments, and wrote the manuscript S.K. and H.C. analyzed the data. I. M.-J. supervised the study, and reviewed and edited the manuscript.

This work was supported by grants from the National Research Foundation [2015R1A2A1A05001794, 2014M3C7A1046047, 2015M3C7A1028790 and the Medical Research Center (2012R1A5A2A44671346)]; Protein metabolism medical research center through Seoul National University Nobel Laureates Invitation program; Brain Korea 21 Plus program for I. M-J. All authors read and approved the final manuscript.

Received: 12 May 2015 Accepted: 23 October 2015

Published online: 31 October 2015

\section{References}

1. Walsh DM, Selkoe DJ. Deciphering the molecular basis of memory failure in Alzheimer's disease. Neuron. 2004:44:181-93.

2. Yankner BA. Mechanisms of neuronal degeneration in Alzheimer's disease. Neuron. 1996:16:921-32.

3. Gouras GK, Tsai J, Naslund J, Vincent B, Edgar M, Checler F, et al. Intraneuronal Abeta42 accumulation in human brain. Am J Pathol. 2000;156:15-20.

4. Selkoe DJ. Cell biology of protein misfolding: the examples of Alzheimer's and Parkinson's diseases. Nat Cell Biol. 2004;6:1054-61.

5. Edbauer D, Winkler E, Regula JT, Pesold B, Steiner H, Haass C. Reconstitution of gamma-secretase activity. Nat Cell Biol. 2003;5:486-8.

6. Vassar R, Bennett BD, Babu-Khan S, Kahn S, Mendiaz EA, Denis P, et al. Betasecretase cleavage of Alzheimer's amyloid precursor protein by the transmembrane aspartic protease BACE. Science. 1999;286:735-41.

7. Iwata N, Tsubuki S, Takaki Y, Shirotani K, Lu B, Gerard NP, et al. Metabolic regulation of brain Abeta by neprilysin. Science. 2001;292:1550-2.

8. Farris W, Mansourian S, Chang Y, Lindsley L, Eckman EA, Frosch MP, et al. Insulin-degrading enzyme regulates the levels of insulin, amyloid betaprotein, and the beta-amyloid precursor protein intracellular domain in vivo. Proc Natl Acad Sci U S A. 2003;100:4162-7. 
9. Yan $\mathrm{P}, \mathrm{Hu} X$, Song $H$, Yin K, Bateman RJ, Cirrito JR, et al. Matrix metalloproteinase-9 degrades amyloid-beta fibrils in vitro and compact plaques in situ. J Biol Chem. 2006;281:24566-74.

10. Vekrellis K, Ye Z, Qiu WQ, Walsh D, Hartley D, Chesneau V, et al. Neurons regulate extracellular levels of amyloid beta-protein via proteolysis by insulin-degrading enzyme. J Neurosci. 2000;20:1657-65.

11. Zhao J, Li L, Leissring MA. Insulin-degrading enzyme is exported via an unconventional protein secretion pathway. Mol Neurodegener. 2009;4:4

12. Mizushima N, Levine B, Cuervo AM, Klionsky DJ. Autophagy fights disease through cellular self-digestion. Nature. 2008;451:1069-75.

13. Frake RA, Ricketts T, Menzies FM, Rubinsztein DC. Autophagy and neurodegeneration. J Clin Invest. 2015;125:65-74.

14. Klionsky DJ. Autophagy: from phenomenology to molecular understanding in less than a decade. Nat Rev Mol Cell Biol. 2007:8:931-7.

15. Son SM, Song H, Byun J, Park KS, Jang HC, Park YJ, et al. Accumulation of autophagosomes contributes to enhanced amyloidogenic APP processing under insulin-resistant conditions. Autophagy. 2012;8:1842-4.

16. Subramani S, Malhotra V. Non-autophagic roles of autophagy-related proteins. EMBO Rep. 2013:14:143-51.

17. Dupont N, Jiang S, Pilli M, Ornatowski W, Bhattacharya D, Deretic V. Autophagy-based unconventional secretory pathway for extracellular delivery of IL-1 beta. EMBO J. 2011;30:4701-11.

18. Torisu T, Torisu K, Lee IH, Liu J, Malide D, Combs CA, et al. Autophagy regulates endothelial cell processing, maturation and secretion of von Willebrand factor. Nat Med. 2013;19:1281-7.

19. Martins I, Wang Y, Michaud M, Ma Y, Sukkurwala $A Q$, Shen S, et al. Molecular mechanisms of ATP secretion during immunogenic cell death. Cell Death Differ. 2014;21:79-91.

20. Nilsson P, Loganathan K, Sekiguchi M, Matsuba Y, Hui K, Tsubuki S, et al. Abeta secretion and plaque formation depend on autophagy. Cell Rep. 2013;5:61-9.

21. Howland DS, Trusko SP, Savage MJ, Reaume AG, Lang DM, Hirsch JD, et al. Modulation of secreted beta-amyloid precursor protein and amyloid betapeptide in brain by cholesterol. J Biol Chem. 1998;273:16576-82.

22. Park $\mid \mathrm{H}$, Hwang EM, Hong HS, Boo JH, Oh SS, Lee J, et al. Lovastatin enhances Abeta production and senile plaque deposition in female Tg2576 mice. Neurobiol Aging. 2003;24:637-43.

23. Wolozin B, Kellman W, Ruosseau P, Celesia GG, Siegel G. Decreased prevalence of Alzheimer disease associated with 3-hydroxy-3-methyglutaryl coenzyme A reductase inhibitors. Arch Neurol. 2000;57:1439-43.

24. Tamboli IY, Barth E, Christian L, Siepmann M, Kumar S, Singh S, et al. Statins promote the degradation of extracellular amyloid \{beta\}-peptide by microglia via stimulation of exosome-associated insulin-degrading enzyme (IDE) secretion. J Biol Chem. 2010;285:37405-14.

25. Zhou Y, Suram A, Venugopal C, Prakasam A, Lin S, Su Y, et al. Geranylgeranyl pyrophosphate stimulates gamma-secretase to increase the generation of Abeta and APP-CTFgamma. FASEB J. 2008;22:47-54.

26. Ostrowski SM, Wilkinson BL, Golde TE, Landreth G. Statins reduce amyloidbeta production through inhibition of protein isoprenylation. J Biol Chem. 2007:282:26832-44.

27. Cole SL, Vassar R. Isoprenoids and Alzheimer's disease: a complex relationship. Neurobiol Dis. 2006;22:209-22.

28. Dorfman VB, Pasquini L, Riudavets M, Lopez-Costa JJ, Villegas A, Troncoso JC, et al. Differential cerebral deposition of IDE and NEP in sporadic and familial Alzheimer's disease. Neurobiol Aging. 2010;31:1743-57.

29. Ma L, Niknejad N, Gorn-Hondermann I, Dayekh K, Dimitroulakos J. Lovastatin induces multiple stress pathways including LKB1/AMPK activation that regulate its cytotoxic effects in squamous cell carcinoma cells. PLoS One. 2012;7:e46055.

30. Kimura N, Tokunaga C, Dalal S, Richardson C, Yoshino K, Hara K, et al. A possible linkage between AMP-activated protein kinase (AMPK) and mammalian target of rapamycin (mTOR) signalling pathway. Genes Cells. 2003;8:65-79.

31. Son SM, Jung ES, Shin HJ, Byun J, Mook-Jung I. Abeta-induced formation of autophagosomes is mediated by RAGE-CaMKKbeta-AMPK signaling. Neurobiol Aging. 2012:33:1006 e1011-1023.

32. Hawley SA, Boudeau J, Reid JL, Mustard KJ, Udd L, Makela TP, et al. Complexes between the LKB1 tumor suppressor, STRAD alpha/beta and MO25 alpha/beta are upstream kinases in the AMP-activated protein kinase cascade. J Biol. 2003;2:28

33. Woods A, Dickerson K, Heath R, Hong SP, Momcilovic M, Johnstone SR, et al. $\mathrm{Ca} 2+$ /calmodulin-dependent protein kinase kinase-beta acts upstream of AMP-activated protein kinase in mammalian cells. Cell Metab. 2005;2:21-33.
34. Eroglu C, Barres BA. Regulation of synaptic connectivity by glia. Nature. 2010:468:223-31.

35. Serrano-Pozo A, Mielke ML, Gomez-Isla T, Betensky RA, Growdon JH, Frosch MP, et al. Reactive glia not only associates with plaques but also parallels tangles in Alzheimer's disease. Am J Pathol. 2011;179:1373-84.

36. Mathur R, Ince PG, Minett T, Garwood CJ, Shaw PJ, Matthews FE, et al. A reduced astrocyte response to beta-amyloid plaques in the ageing brain associates with cognitive impairment. PLoS One. 2015;10, e0118463.

37. Thal DR. The role of astrocytes in amyloid beta-protein toxicity and clearance. Exp Neurol. 2012;236:1-5.

38. Xiao Q, Yan P, Ma X, Liu H, Perez R, Zhu A, et al. Enhancing astrocytic lysosome biogenesis facilitates Abeta clearance and attenuates amyloid plaque pathogenesis. J Neurosci. 2014:34:9607-20.

39. Yin KJ, Cirrito JR, Yan P, Hu X, Xiao Q, Pan X, et al. Matrix metalloproteinases expressed by astrocytes mediate extracellular amyloid-beta peptide catabolism. J Neurosci. 2006;26:10939-48.

40. Oiu WQ Walsh DM, Ye Z, Vekrellis K, Zhang J, Podlisny MB, et al. Insulindegrading enzyme regulates extracellular levels of amyloid beta-protein by degradation. J Biol Chem. 1998;273:32730-8.

41. Haag MD, Hofman A, Koudstaal PJ, Stricker BH, Breteler MM. Statins are associated with a reduced risk of Alzheimer disease regardless of lipophilicity. The Rotterdam Study. J Neurol Neurosurg Psychiatry. 2009;80:13-7.

42. Kandiah N, Feldman HH. Therapeutic potential of statins in Alzheimer's disease. J Neurol Sci. 2009;283:230-4.

43. Yang DS, Stavrides P, Mohan PS, Kaushik S, Kumar A, Ohno M, et al. Reversal of autophagy dysfunction in the TgCRND8 mouse model of Alzheimer's disease ameliorates amyloid pathologies and memory deficits. Brain. 2011:134:258-77.

44. Nixon RA. The role of autophagy in neurodegenerative disease. Nat Med. 2013;19:983-97.

45. Turner AJ, Fisk L, Nalivaeva NN. Targeting amyloid-degrading enzymes as therapeutic strategies in neurodegeneration. Ann N Y Acad Sci. 2004;1035:1-20.

46. Son SM, Nam DW, Cha MY, Kim KH, Byun J, Ryu H, et al. Thrombospondin-1 prevents amyloid beta-mediated synaptic pathology in Alzheimer's disease. Neurobiol Aging. 2015

47. Son SM, Byun J, Roh SE, Kim SJ, Mook-Jung I. Reduced IRE1alpha mediates apoptotic cell death by disrupting calcium homeostasis via the InsP3 receptor. Cell Death Dis. 2014;5:e1188.

48. Son SM, Song H, Byun J, Park KS, Jang HC, Park YJ, et al. Altered APP processing in insulin-resistant conditions is mediated by autophagosome accumulation via the inhibition of mammalian target of rapamycin pathway. Diabetes. 2012:61:3126-38

49. Byun J, Son SM, Cha MY, Shong M, Hwang YJ, Kim Y, et al. CR6-interacting factor 1 is a key regulator in Abeta-induced mitochondrial disruption and pathogenesis of Alzheimer's disease. Cell Death Differ. 2015;22(6):959-73. http://www.ncbi.n/m.nih.gov/pubmed/?term=CR6-interacting+factor+1+is+a +key+regulator+in+Abetainduced+mitochondrial+disruption+and +pathogenesis+of+Alzheimer\%27s+disease

50. Trajkovic K, Hsu C, Chiantia S, Rajendran L, Wenzel D, Wieland F, et al. Ceramide triggers budding of exosome vesicles into multivesicular endosomes. Science. 2008:319:1244-7.

51. Santuccione AC, Merlini M, Shetty A, Tackenberg C, Bali J, Ferretti MT, et al. Active vaccination with ankyrin $\mathrm{G}$ reduces beta-amyloid pathology in APP transgenic mice. Mol Psychiatry. 2013;18:358-68.

52. Choi I, Kim B, Byun JW, Baik SH, Huh YH, Kim JH, et al. LRRK2 G2019S mutation attenuates microglial motility by inhibiting focal adhesion kinase. Nat Commun. 2015:6:8255. 